\title{
ANÁLISE ESTRUTURAL DE CICLODEXTRINAS: UM ESTUDO COMPARATIVO ENTRE MÉTODOS TEÓRICOS CLÁSSICOS E QUÂNTICOS
}

\author{
Marta A. F. O. Britto, Clebio S. Nascimento Jr. e Hélio F. dos Santos* \\ Departamento de Química, Instituto de Ciências Exatas, Universidade Federal de Juiz de Fora, Campus Universitário Martelos,
} 36036-330 Juiz de Fora - MG

Recebido em 3/9/03; aceito em 23/3/04; publicado na web em 27/07/04

\begin{abstract}
STRUCTURAL ANALYSIS OF CYCLODEXTRINS: A COMPARATIVE STUDY OF CLASSICAL AND QUANTUM MECHANICAL METHODS. In the present work, we analyzed the accuracy of distinct theoretical methods to reproduce the solid state structures of cyclodextrins. The $\alpha, \beta$ and $\gamma$-cyclodextrins (CD) were considered and also their hydrates with included water molecules: $\alpha$-CD. $2 \mathrm{H}_{2} \mathrm{O}, \beta-\mathrm{CD} \cdot 10 \mathrm{H}_{2} \mathrm{O}$ and $\gamma-\mathrm{CD} \cdot 12 \mathrm{H}_{2} \mathrm{O}$. The geometries were fully optimized using Molecular Mechanics (MM2), semiempirical (AM1 and PM3) and ab initio (HF/3-21G) methods and quantitatively compared with experimental data from X ray diffraction. The results obtained from the classical MM2 method were in best agreement with the experiment. The semiempirical and $a b$ initio structures were also in satisfactory accordance with the experimental data. In general, the PM3 method was found to be more suitable than the AM1 to describe the CD geometries, mainly when the intramolecular hydrogen bonds are considered.
\end{abstract}

Keywords: cyclodextrin; quantum-mechanical calculation; molecular mechanics.

\section{INTRODUÇÃO}

Ciclodextrinas (CD’s) são oligossacarídeos cíclicos formados por moléculas de D-glicose unidas através de ligações glicosídicas $\alpha(1-4)$ (Figura 1a), obtidas a partir da degradação enzimática do amido ${ }^{1}$. As CD's mais conhecidas são as $\alpha, \beta$ e $\gamma$-ciclodextrinas, constituídas por 6,7 e 8 unidades de glicose, respectivamente, que adotam a conformação de cadeira. Do ponto de vista estrutural, as CD's apresentam-se na forma de "cones truncados" com o lado mais largo formado pelas hidroxilas secundárias em C-2 e C-3 e a face mais estreita constituída pelas hidroxilas primárias ligadas em C-6 (Figura 1b). A dimensão da cavidade é determinada pelo número de unidades de glicose constituintes da $\mathrm{CD}$. Os átomos de oxigênio envolvidos nas ligações glicosídicas (em C-1 e C-4) e os átomos de hidrogênio ligados em C3 e C-5 determinam o caráter hidrofóbico do interior da cavidade das CD's (Figura 1b). A presença das hidroxilas livres na parte externa das CD's confere a essas moléculas um caráter hidrofílico. Esse arranjo estrutural das moléculas de glicose nas CD's possibilita a utilização desses compostos como hospedeiros na formação de complexos de inclusão. A presença de uma cavidade hidrofóbica e de grupos hidroxilas livres na parte externa da molécula permite a "dissolução" em meio aquoso de compostos (hóspedes) de baixa solubilidade. Esse aspecto molecular tem possibilitado a utilização de ciclodextrinas em diferentes áreas da ciência e tecnologia, sendo o principal domínio de aplicação a indústria farmacêutica, em função da possibilidade de obtenção de novos fármacos com propriedades físicas e químicas diferentes e o mesmo princípio ativo.

Apesar do grande número de trabalhos desenvolvidos na linha de pesquisa de compostos de inclusão com CD's, a caracterização dos complexos constitui ainda hoje uma etapa delicada no processo de síntese. A fraca interação entre hóspede e hospedeiro representa uma característica intrínseca do sistema molecular, a qual dificulta a utilização de algumas técnicas analíticas aplicadas à solução. Nesse contexto, a química computacional vem contribuindo de forma sig-

*e-mail: helius@quimica.ufjf.br
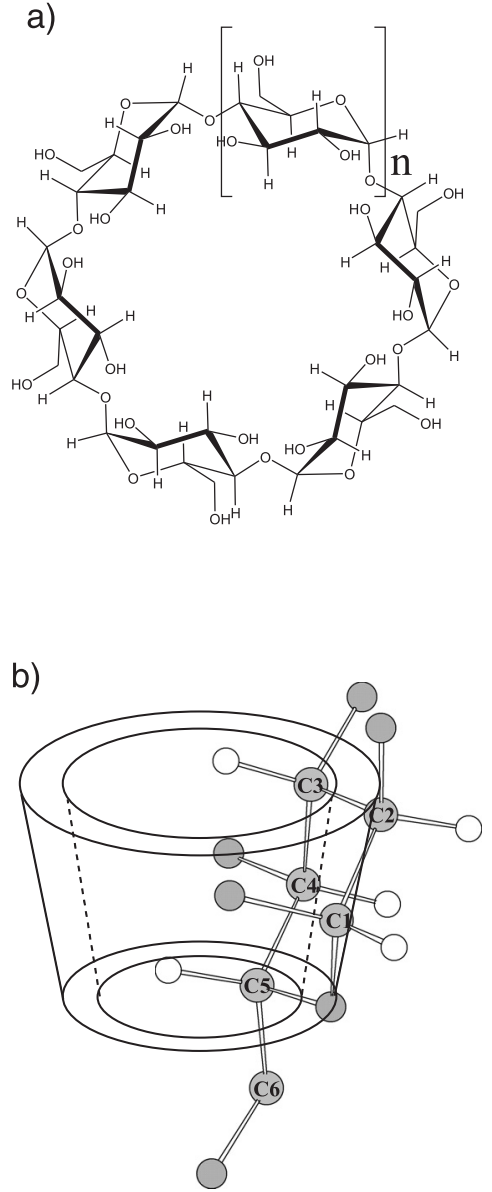

Figura 1. (a) Estrutura geral das ciclodextrinas (CD's). Os derivados $\alpha, \beta$ e $\gamma$-CD são definidos por $n=1,2$ e 3, respectivamente. (b) Representação esquemática da estrutura tridimensional das CD's, mostrando as características estruturais definidas pelo arranjo das unidades de glicose 
nificativa para a determinação de estruturas e propriedades físicoquímicas de ciclodextrinas e seus complexos de inclusão.

O primeiro estudo computacional envolvendo ciclodextrinas foi publicado em 1970 por Sundararajan e $\mathrm{Rao}^{2}$. Nesse período, somente a estrutura da $\alpha-C D$ era conhecida e os pesquisadores estavam interessados em usar as ferramentas da química computacional para a determinação das conformações das unidades de glicose constituintes do macrociclo. O método computacional empregado nesse estudo é o que chamamos hoje de Mecânica Molecular (MM), porém com várias aproximações relacionadas aos tipos de funções potenciais implementadas no campo de força.

Em outro estudo, French e Murphy ${ }^{3}$ correlacionaram distâncias de "ligações virtuais" com mudanças geométricas nas unidades de glicose da $\alpha$-CD. Cada resíduo de glicose foi considerado como um corpo rígido unido por uma "ligação virtual", que foi definida como um vetor que se estendia do O-4 de um resíduo de glicose até O-1 de um resíduo adjacente. Assim, os autores analisaram a existência de parâmetros estruturais que definiam, de forma adequada, a geometria da $\alpha-C D$. Posteriormente, French e Murphy derivaram esse conjunto de parâmetros para a $\beta$ e $\gamma$-CD's e, usando essa aproximação, fizeram relevantes argumentações sobre as inter-relações das estruturas dos monômeros e suas orientações relativas no macrociclo ${ }^{4}$.

Mais recentemente, em 1994, Jicsinszky e Bakó 5 realizaram cálculos quânticos, utilizando o método semi-empírico AM1, para estudarem as estruturas da $\alpha, \beta$ e $\gamma$-CD. O objetivo dos autores era obter uma distribuição de cargas confiável para as unidades de glicose presentes nas CD's. Além disso, buscavam informações quantitativas para a descrição das geometrias das respectivas CD's. Para isso, foram realizados cálculos da distribuição de cargas e de vários parâmetros estruturais. Os resultados mostraram um acordo satisfatório entre as geometrias obtidas através dos cálculos quânticos e as estruturas obtidas no estado sólido, sendo as distribuições de cargas similares para todas as unidades de glicose, o que demonstrava a simetria dessas moléculas.

Em outro trabalho, Yannakopoulou e colaboradores ${ }^{6}$ realizaram cálculos semi-empíricos usando o metódo AM1 para estudar a estrutura da $\beta-C D$ e dos seus respectivos complexos de inclusão formados com os substratos 1,7-dioxaspiro[5,5] undecano e nonanal. Os autores concluíram que o metódo AM1 fornece resultados satisfatórios para os processos de inclusão envolvendo a $\beta$-CD.

Em 1998, um artigo completo de revisão sobre a aplicação de química computacional em estudos com ciclodextrinas foi publicado por Lipkowitz ${ }^{7}$. Nesse estudo, o autor relata um número significativo de aplicações de metodologias teóricas, que têm sido direcionadas para uma melhor descrição da estrutura das CD's. Em trabalho recente, Liu e $\mathrm{Li}^{8}$ utilizaram metodologias teóricas para fazer uma análise estrutural da $\beta$-CD. Eles fizeram um estudo comparativo de dados cristalográficos com estruturas obtidas através de cálculos quânticos. Os autores mostraram através da análise de parâmetros estruturais que o método semi-empírico PM3 descreve melhor a estrutura da $\beta$-CD quando comparado com o método AM1. O método AM1 mostrou-se ineficiente na medida que forneceu geometrias bastante distorcidas para a estrutura da $\beta-\mathrm{CD}$, devido principalmente à descrição incorreta de ligações de hidrogênio, diferentemente do método PM3. Em 2002, Bodor e Buchwald ${ }^{9}$ realizaram um estudo teórico com o objetivo de investigar aspectos físico-químicos relevantes para a formação de vários complexos de inclusão com ciclodextrinas. Nesse estudo, os autores buscaram relações entre a estrutura e a estabilidade desses complexos. Uma análise estatística envolvendo vários parâmetros, como tamanho molecular, hidrofobicidade, ângulos de ligação e propriedades eletrônicas, foi realizada. Os resultados mostraram a importância de alguns grupos funcionais presentes nos substratos para a estabilidade dos complexos de inclusão formados. Outros trabalhos utilizando metodologias teóricas e computacionais direcionados ao estudo estrutural de ciclodextrinas podem ser encontrados nas referências ${ }^{10-13}$.

No presente trabalho, uma análise estrutural sistemática das principais ciclodextrinas ( $\alpha, \beta$ e $\gamma$-CD) foi realizada utilizando métodos clássicos de mecânica molecular (MM2) e quânticos semi-empíricos (AM1 e PM3) e ab initio (HF/3-21G). O objetivo deste estudo é avaliar a qualidade das geometrias obtidas através de diferentes níveis de teoria na descrição estrutural dessa classe de moléculas.

\section{METODOLOGIA DE CÁLCULO}

As estruturas das $\alpha, \beta$ e $\gamma$-ciclodextrinas foram obtidas a partir de coordenadas cartesianas disponíveis em banco de dados de estruturas cristalográficas ${ }^{14}$. As estruturas obtidas apresentaram-se na forma de hidratos $\alpha$-CD. $6 \mathrm{H}_{2} \mathrm{O}, \beta-\mathrm{CD} .15 \mathrm{H}_{2} \mathrm{O}$ e $\gamma$-CD. $18 \mathrm{H}_{2} \mathrm{O}$, contendo 2,10 e 12 moléculas de água no interior da cavidade das respectivas CD's. Segundo Connors ${ }^{15}$, a $\alpha$-CD é geralmente encontrada na forma hexa-hidratada ${ }^{16}$, a $\beta$-CD existe usualmente na forma undecahidratada ${ }^{17}$ ou dodeca-hidratada ${ }^{18}$, dependendo da umidade relativa, e a $\gamma$-CD é, em algumas situações, encontrada como sendo octahidratada, podendo ser cristalizada com 7 a 18 moléculas de água ${ }^{19}$.

As estruturas cristalográficas das CD's e as moléculas de água presentes nessas estruturas foram obtidas sem os átomos de hidrogênio. Esses átomos foram adicionados aleatoriamente, utilizando-se o programa computacional Spartan-Pro ${ }^{20}$. As conformações das hidroxilas primárias e secundárias foram modificadas visando favorecer a formação de ligações de hidrogênio intramoleculares, com o intuído de conferir um caráter mais rígido às estruturas das CD's. As geometrias obtidas serviram como ponto de partida para as otimizações de geometria realizadas através de diferentes metodologias teóricas clássica (MM2), semi-empíricas (AM1 e PM3) e ab initio (HF/3-21G). Todas as estruturas foram completamente otimizadas e caracterizadas como mínimos verdadeiros na Superfície de Energia Potencial (PES) através da análise das freqüências harmônicas, sendo todas as freqüências calculadas reais. Os cálculos quânticos foram realizados utilizando o programa GAUSSIAN $/ 98^{21}$ e os cálculos de Mecânica Molecular foram realizados no programa computacional CHEM-3D 22 .

Com o objetivo de compararmos quantitativamente as estruturas obtidas a partir dos cálculos clássicos e quânticos, acima relacionados, com as estruturas cristalográficas da $\alpha, \beta$ e $\gamma$-CD, foram feitas medidas de vários parâmetros estruturais, incluindo ângulos diedros, ângulos de ligação e distâncias entre os oxigênios de hidroxilas secundárias de unidades de glicose adjacentes nas respectivas CD's.

Todos os parâmetros estruturais utilizados são definidos na Figura 2a. Os ângulos $\Phi, \Psi$ e E são ângulos de torção que descrevem a conformação molecular em relação às ligações glicosídicas, sendo que E também está relacionado com a abertura da cavidade. $\Omega$ e $\Xi$ descrevem a orientação das hidroxilas primárias em relação ao anel piranosídico. Os ângulos $\Gamma, \mathrm{T}, \Theta$ e $\Pi$ descrevem a torção de cada unidade de glicose. $\mathrm{K}$ e Y descrevem a torção das hidroxilas secundárias e os ângulos $\vartheta, \Lambda$ e Z são ângulos diedros relativos às ligações glicosídicas. Também foi analisado o ângulo $\alpha$, definido como o ângulo entre três oxigênios anoméricos consecutivos $\left(\alpha:\left[\mathrm{O}_{4} \mathrm{O}_{4}, \mathrm{O}_{4}\right.\right.$, , $]$, que permite avaliar a abertura da cavidade, e o ângulo $\beta$ que é um ângulo relacionado com as ligações de hidrogênio intramoleculares das CD's $(\beta:[\mathrm{OOH}])$.

Os efeitos das águas de inclusão nas estruturas das CD's foram analisados nos níveis MM2, AM1 e PM3, considerando as geometrias dos hidratos $\alpha$-CD. $2 \mathrm{H}_{2} \mathrm{O}, \beta-\mathrm{CD} .10 \mathrm{H}_{2} \mathrm{O}$ e $\gamma-\mathrm{CD} .12 \mathrm{H}_{2} \mathrm{O}$.

Cálculos do momento de dipolo elétrico $(\mu)$ das CD's foram também realizados no nível HF/3-21G, considerando as estruturas 
a)
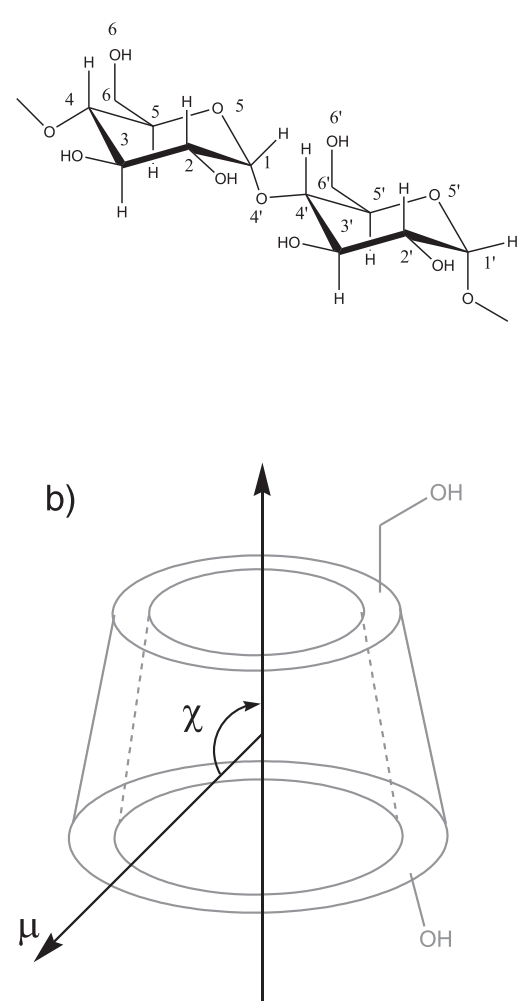

Figura 2. (a) Representação esquemática de duas unidades de glicose da ciclodextrina com a numeração de seus átomos. Ângulos diedros:

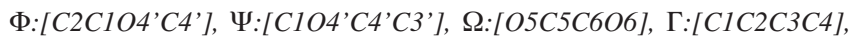
$\mathrm{T}:[C 3 C 4 C 5 O 5], \mathrm{K}:[O 2 C 2 C 1 O 5], \mathrm{Y}:[O 3 C 3 C 4 C 5], \Theta:[C 2 C 3 C 4 C 5]$, П:[C4C5O5C1], $\Xi:[C 4 C 5 C 606], \vartheta:[04 C 4 C 5 O 5], \mathrm{Z}:[04 C 4 C 5 C 6]$, $\Lambda:[O 4$ 'C1O5C5], E:[O4'C1C4C5]. Ângulo de ligação: $\theta:$ [C1O4'C4']. Outros ângulos: $\alpha$ : [O4O4'O4"] e $\beta$ : [OOH]. (b) Definição do ângulo de orientação $(\chi)$ do momento de dipolo elétrico $(\mu)$ para as moléculas de ciclodextrinas otimizadas através dos métodos quânticos AM1, PM3 e HF/3-21G. $\mathrm{O}$ módulo e a orientação do dipolo (Figura 2 b) foram considerados nessa análise, visando avaliar os efeitos das distorções moleculares nas propriedades eletrônicas das ciclodextrinas.

\section{RESULTADOS E DISCUSSÃO}

Inicialmente, as geometrias da $\alpha, \beta$ e $\gamma$-CD foram otimizadas utilizando o método empírico de Mecânica Molecular (MM2). As estruturas otimizadas no nível MM2 serviram como ponto de partida para as subseqüentes otimizações, realizadas através de métodos quânticos semi-empíricos (AM1 e PM3) e ab-initio (HF/3-21G).

Nas Tabelas 1, 2 e 3 são listados os ângulos médios obtidos das estruturas livres e hidratadas das ciclodextrinas. Os valores apresentados referem-se à média aritmética dos respectivos ângulos de cada uma das unidades glicosídicas. Além dos valores médios, são também apresentados os desvios padrão para cada um dos parâmetros relacionados, juntamente com os dados experimentais obtidos da estrutura cristalográfica. Os desvios padrão apresentados nas Tabelas 1-3 fornecem informações sobre a flexibilidade conformacional do grupo envolvido na torção definida pelo parâmetro estrutural. Analisando os valores da Tabela 1, referentes aos parâmetros estruturais calculados para $\alpha$-CD e $\alpha$-CD. $2 \mathrm{H}_{2} \mathrm{O}$, é possível verificar um desvio padrão relativamente alto $\left(\sim 20^{\circ}\right)$ no ângulo $\Psi$ em todos os níveis de teoria, o que mostra uma maior flexibilidade na conformação relativa das unidades de glicose. Esse comportamento é também observado na estrutura do estado sólido, mostrando que a estrutura da $\alpha-C D$ não é perfeitamente simétrica. Nota-se também um desvio padrão significativamente alto nos ângulos $\Xi$ e $\Omega$, demonstrando uma alta flexibilidade na conformação das hidroxilas primárias. Um aspecto interessante dos resultados obtidos, é que não ocorrem alterações significativas nos valores dos ângulos médios com a inclusão das moléculas de água no interior da cavidade da $\alpha-C D$. Analisando-se as Tabelas 2 e 3, observam-se resultados análogos àqueles obtidos para a $\alpha-C D$, relacionados, principalmente, aos altos valores no desvio padrão dos ângulos $\Xi$ e $\Omega$, e ao fato de que os cálculos considerando as águas de inclusão não levam a distorções significativas na estrutura molecular.

Tabela 1. Parâmetros estruturais (em graus) calculados para a $\alpha-\mathrm{CD}$ e $\alpha$-CD.2 $\mathrm{H}_{2} \mathrm{O}$ (entre colchetes), utilizando diferentes métodos teóricos. Os valores médios para cada ângulo, considerando as 6 unidades de glicose, são apresentados juntamente com os respectivos desvios padrão

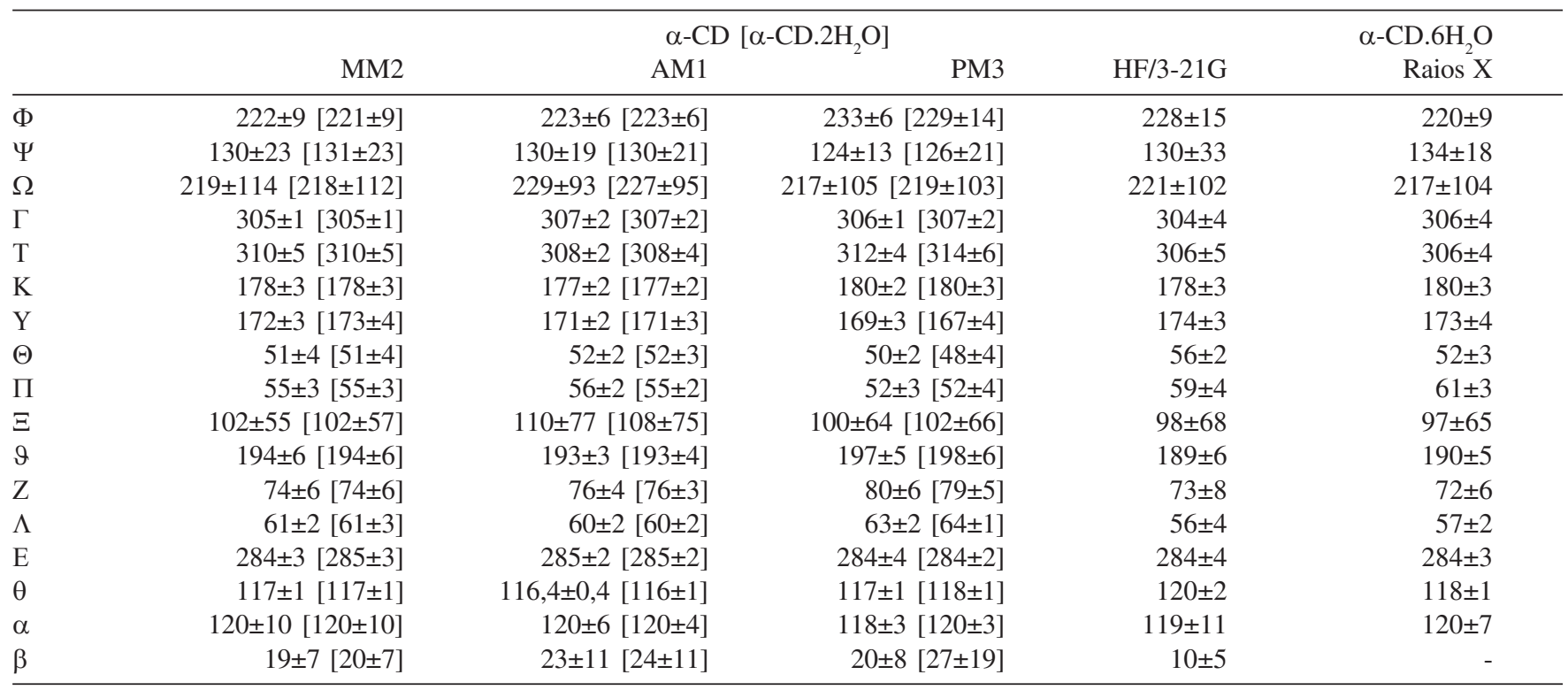


Tabela 2. Parâmetros estruturais (em graus) calculados para a $\beta-\mathrm{CD}$ e $\beta-\mathrm{CD} \cdot 10 \mathrm{H}_{2} \mathrm{O}$ (entre colchetes), utilizando diferentes métodos teóricos. Os valores médios para cada ângulo, considerando as 7 unidades de glicose, são apresentados juntamente com os respectivos desvios padrão

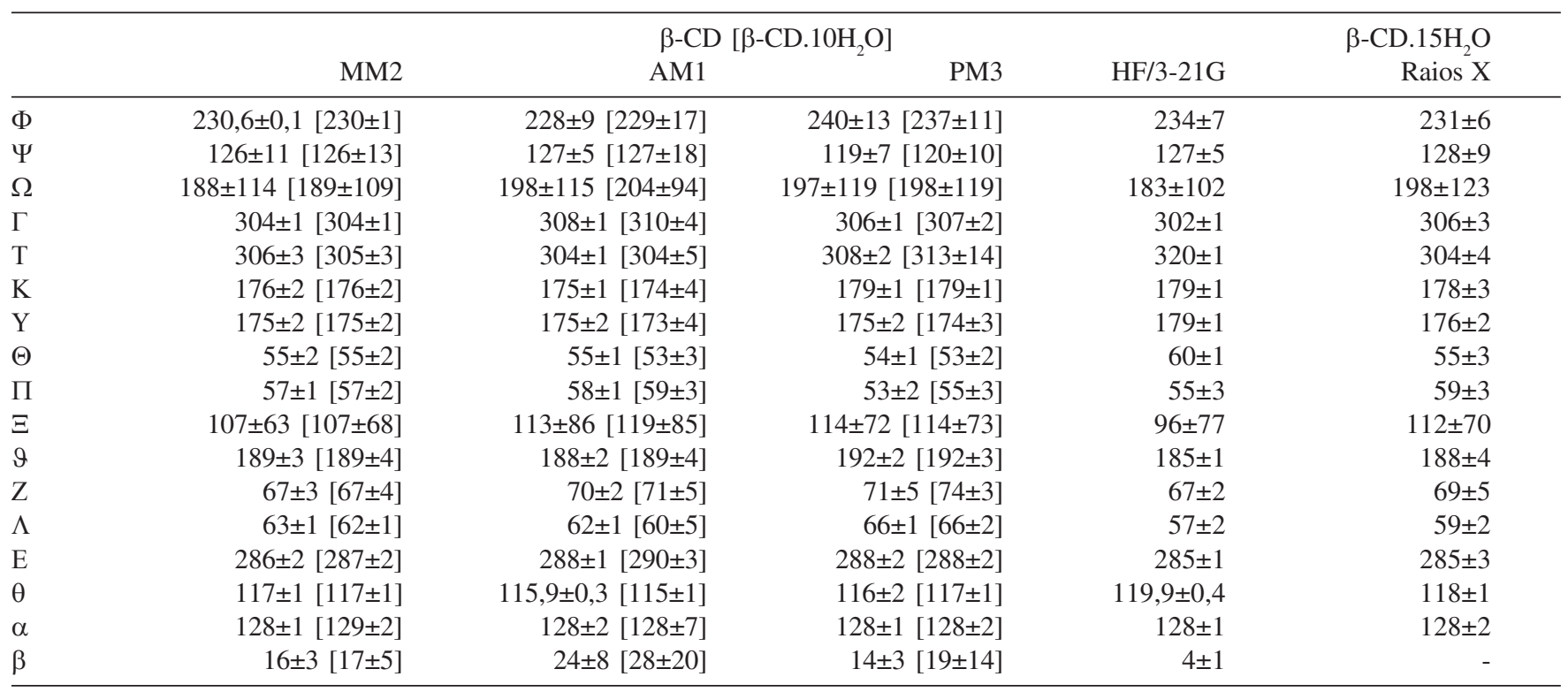

Tabela 3. Parâmetros estruturais (em graus) calculados para a $\gamma$-CD e $\gamma$-CD.12 $\mathrm{H}_{2} \mathrm{O}$ (entre colchetes), utilizando diferentes métodos teóricos. Os valores médios para cada ângulo, considerando as 8 unidades de glicose, são apresentados juntamente com os respectivos desvios padrão

\begin{tabular}{|c|c|c|c|c|c|}
\hline & \multicolumn{4}{|c|}{$\gamma-\mathrm{CD}\left[\gamma-\mathrm{CD} .12 \mathrm{H}_{2} \mathrm{O}\right]$} & \multirow{2}{*}{$\begin{array}{r}\gamma-\mathrm{CD} .18 \mathrm{H}_{2} \mathrm{O} \\
\text { Raios X }\end{array}$} \\
\hline & MM2 & AM1 & PM3 & $\mathrm{HF} / 3-21 \mathrm{G}$ & \\
\hline $\bar{\Phi}$ & $231 \pm 9[230 \pm 15]$ & $231 \pm 11[229 \pm 15]$ & $241 \pm 12[239 \pm 7]$ & $241 \pm 16$ & $231 \pm 6$ \\
\hline$\Omega$ & $203 \pm 116[206 \pm 112]$ & $218 \pm 97$ [217士97] & $211 \pm 107[211 \pm 102]$ & $206 \pm 111$ & $214 \pm 110$ \\
\hline$\Gamma$ & $304 \pm 4[306 \pm 2]$ & $308 \pm 2[310 \pm 4]$ & $307 \pm 3[305 \pm 2]$ & $302 \pm 2$ & $307 \pm 4$ \\
\hline $\mathrm{T}$ & $302 \pm 3[303 \pm 3]$ & $301 \pm 4[303 \pm 8]$ & $305 \pm 3[303 \pm 3]$ & $303 \pm 3$ & $301 \pm 4$ \\
\hline$\Theta$ & $57 \pm 3[55 \pm 3]$ & $56 \pm 2[54 \pm 8]$ & $55 \pm 3[58 \pm 2]$ & $59 \pm 2$ & $57 \pm 3$ \\
\hline$\Pi$ & $58 \pm 3[59 \pm 2]$ & $58 \pm 1[59 \pm 3]$ & $54 \pm 3[55 \pm 2]$ & $59 \pm 3$ & $62 \pm 3$ \\
\hline$\Xi$ & $100 \pm 58[104 \pm 63]$ & $113 \pm 78[112 \pm 78]$ & $107 \pm 66[108 \pm 71]$ & $98 \pm 65$ & $107 \pm 64$ \\
\hline$\vartheta$ & $185 \pm 3[186 \pm 3]$ & $185 \pm 2[187 \pm 8]$ & $188 \pm 3[187 \pm 4]$ & $184 \pm 3$ & $184 \pm 3$ \\
\hline $\mathrm{Z}$ & $65 \pm 3[66 \pm 4]$ & $68 \pm 3[69 \pm 8]$ & $71 \pm 4[68 \pm 4]$ & $68 \pm 4$ & $69 \pm 5$ \\
\hline$\beta$ & $18 \pm 4[42 \pm 21]$ & $27 \pm 11[31 \pm 20]$ & $14 \pm 5[22 \pm 7]$ & $9 \pm 5$ & - \\
\hline
\end{tabular}

Um outro parâmetro molecular importante para a caracterização da estrutura das ciclodextrinas é o ângulo $\alpha$, definido entre três oxigênios glicosídicos adjacentes. Esse parâmetro estrutural representa a regularidade do polígono formado pelos oxigênios glicosídicos e pode ser relacionado ao diâmetro interno da cavidade. Para a $\alpha-C D$, o valor esperado é de $120^{\circ}$, correspondendo a um hexágono regular, sendo o valor médio calculado igual a $118 \pm 3^{\circ}$ (PM3). Para a $\beta$ e $\gamma$ $\mathrm{CD}$ os valores obtidos teoricamente foram $128 \pm 1^{\circ}$ e $134 \pm 1^{\circ}$, respectivamente (valores PM3). Os valores esperados são $128^{\circ}$ e $134^{\circ}$, sugerindo que o diâmetro interno da cavidade não sofre alteração considerável em nenhuma das geometrias analisadas. A variação no ângulo $\alpha$ é também pequena quando as águas de inclusão são consideradas. A análise desse parâmetro é interessante dentro do contexto da formação de complexos de inclusão, sendo que alterações no diâmetro da cavidade podem modificar a termodinâmica desses processos.

Os desvios médios dos ângulos calculados em diferentes níveis de teoria em relação aos dados experimentais são apresentados na Tabela 4. Analisando os resultados pode ser observado que, de forma geral, o método PM3 descreve melhor o ângulo K, relacionado à conformação das hidroxilas secundárias e os ângulos $\Omega$ e $\Xi$, relacionados às posições espaciais das hidroxilas primárias. Para esses ângulos, os desvios obtidos com o método PM3 foram significativamente menores que aqueles calculados considerando outras metodologias teóricas. Para os ângulos $\Phi$ e $\Psi$, que definem a conformação relativa das unidades de glicose, os desvios obtidos com os 
Tabela 4. Desvios médios (em graus) dos ângulos calculados para as estruturas das ciclodextrinas em diferentes níveis de teoria e obtidos a partir da estrutura cristalográfica

\begin{tabular}{|c|c|c|c|c|c|c|c|c|c|c|c|c|}
\hline & \multicolumn{4}{|c|}{$\alpha-C D$} & \multicolumn{4}{|c|}{$\beta-C D$} & \multicolumn{4}{|c|}{$\gamma-\mathrm{CD}$} \\
\hline & MM2 & AM1 & PM3 & $\mathrm{HF}^{\mathrm{a}}$ & MM2 & AM1 & PM3 & $\mathrm{HF}^{\mathrm{a}}$ & MM2 & AM1 & PM3 & $\mathrm{HF}^{\mathrm{a}}$ \\
\hline$\Phi$ & 3,5 & 6,4 & 12,6 & 10,5 & 4,4 & 8,5 & 12,9 & 9,5 & 3,8 & 6,6 & 12,1 & 14,1 \\
\hline$\Psi$ & 6,5 & 4,6 & 12,5 & 11,0 & 5,3 & 10,9 & 11,5 & 5,3 & 4,4 & 10,4 & 10,3 & 15,9 \\
\hline$\Omega$ & 9,9 & 16,2 & 3,8 & 5,5 & 9,4 & 14,1 & 5,8 & 17,4 & 13,0 & 13,4 & 5,8 & 11,3 \\
\hline$\Gamma$ & 2,3 & 2,9 & 2,5 & 5,0 & 2,3 & 2,9 & 2,3 & 3,9 & 1,3 & 2,2 & 3,9 & 5,2 \\
\hline $\mathrm{T}$ & 4,2 & 3,0 & 6,2 & 5,3 & 2,0 & 2,3 & 4,4 & 3,3 & 3,6 & 6,4 & 4,4 & 4,1 \\
\hline $\mathrm{K}$ & 3,2 & 3,1 & 2,4 & 3,1 & 3,0 & 3,9 & 2,1 & 2,2 & 4,1 & 3,1 & 2,9 & 3,7 \\
\hline $\mathrm{Y}$ & 2,7 & 3,2 & 5,1 & 4,6 & 1,3 & 0,9 & 1,5 & 2,7 & 2,7 & 1,2 & 3,2 & 2,2 \\
\hline$\Theta$ & 2,8 & 2,1 & 2,8 & 4,8 & 1,6 & 1,7 & 2,4 & 4,3 & 3,3 & 1,8 & 3,2 & 3,9 \\
\hline$\Pi$ & 5,4 & 4,8 & 8,9 & 4,5 & 2,3 & 2,8 & 6,2 & 2,5 & 4,1 & 4,5 & 7,6 & 4,8 \\
\hline$\Xi$ & 11,1 & 16,2 & 4,8 & 4,6 & 5,1 & 13,3 & 4,0 & 18,3 & 11,5 & 14,7 & 6,2 & 12,3 \\
\hline$\vartheta$ & 4,2 & 3,7 & 6,9 & 5,5 & 1,2 & 2,0 & 4,1 & 3,3 & 2,2 & 2,2 & 4,6 & 3,5 \\
\hline $\mathrm{Z}$ & 3,7 & 4,7 & 8,7 & 5,6 & 2,2 & 2,2 & 4,7 & 3,7 & 2,4 & 3,3 & 5,5 & 4,4 \\
\hline$\Lambda$ & 4,4 & 3,3 & 5,9 & 3,0 & 3,4 & 2,4 & 7,0 & 2,0 & 3,3 & 2,4 & 6,9 & 4,6 \\
\hline $\mathrm{E}$ & 0,9 & 2,3 & 2,9 & 3,8 & 1,3 & 4,2 & 3,3 & 2,3 & 1,1 & 2,5 & 2,6 & 3,8 \\
\hline$\theta$ & 1,1 & 1,5 & 1,1 & 2,6 & 1,0 & 1,6 & 1,5 & 2,3 & 0,8 & 1,6 & 1,1 & 2,2 \\
\hline$\alpha$ & 3,0 & 1,8 & 4,8 & 4,3 & 1,4 & 1,9 & 1,9 & 2,2 & 1,1 & 2,8 & 1,1 & 3,5 \\
\hline
\end{tabular}

${ }^{\mathrm{a} A}$ base de funções utilizada foi 3-21G.

métodos quânticos foram significativos, sendo aqueles calculados no nível PM3 mais pronunciados. A conformação das unidades de glicose é bem descrita em todos os níveis de teoria considerados, como mostrado pelos baixos valores dos desvios dos ângulos $\Gamma, \mathrm{T}$, $\Theta$ e П. Para o ângulo П, relacionado à posição espacial do oxigênio endocíclico, os desvios do método PM3 foram maiores. A conformação ao redor das ligações glicosídicas, definidas pelos ângulos de torção $\vartheta, \Lambda$ e Z, é bem descrita pelos métodos teóricos considerados. Os demais parâmetros estruturais apresentaram desvios médios em relação aos dados cristalográficos menores que $4^{\circ}$.

Na Tabela 5 são apresentados os desvios globais para cada método, descrito pelo RMS ("Root Mean Square"), calculado de acordo com a Equação (1).

$R M S=\sqrt{\frac{1}{16} \sum_{i=1}^{16} d_{i}^{2}} \therefore d_{i}=\frac{1}{n} \sum_{j=1}^{n}\left(D_{j}^{t}-D_{j}^{r x}\right)$

Na equação $(1), n=6(\alpha-C D), 7$ ( $\beta$-CD) e $8(\gamma-C D)$. $D_{j}$ referemse aos parâmetros estruturais teóricos $\left(\mathrm{D}_{\mathrm{j}}^{\mathrm{t}}\right)$ e experimentais $\left(\mathrm{D}_{\mathrm{j}}^{\mathrm{rx}}\right)$. $\mathrm{O}$

Tabela 5. Valores de RMS (em graus) calculados para os diferentes métodos teóricos em relação à estrutura cristalográfica

\begin{tabular}{lcccc}
\hline & \multicolumn{5}{c}{ Valores de RMS em graus } \\
& MM2 & AM1 & PM3 & HF/3-21G \\
& & & & \\
\hline$\alpha-C D$ & $5,1[3,7]$ & $6,6[3,6]$ & $6,6[6,9]$ & $5,7[5,8]$ \\
$\alpha-C D .2 H 2 O$ & $4,5[3,6]$ & $6,1[3,6]$ & $6,7[6,8]$ & - \\
$\beta-C D$ & $4,6[3,9]$ & $6,5[4,6]$ & $5,7[5,8]$ & $7,2[3,7]$ \\
$\beta-C D .10 H 2 O$ & $3,8[2,5]$ & $8,3[6,2]$ & $6,1[5,7]$ & - \\
$\gamma-C D$ & $5,2[3,0]$ & $6,4[4,4]$ & $5,9[5,9]$ & $7,6[6,7]$ \\
$\gamma-C D .12 H 2 O$ & $7,1[6,7]$ & $7,1[5,5]$ & $6,3[5,9]$ & - \\
\hline
\end{tabular}

${ }^{\mathrm{a} O} \mathrm{RMS}$ foi calculado de acordo com a equação (1) considerando os parâmetros estruturais definidos na Figura 2a. Os valores entre colchetes foram calculados excluindo os ângulos $\Xi$ e $\Omega$.
RMS total foi calculado utilizando-se os 16 ângulos medidos e o parcial calculado desconsiderando os ângulos $\Xi$ e $\Omega$, relativos às hidroxilas primárias. O método clássico com campo de força MM2 foi o que apresentou os melhores resultados para as estruturas das ciclodextrinas. Os desvios obtidos para o método AM1 são, em geral, maiores que os respectivos valores calculados para as estruturas obtidas no método PM3, quando o desvio total é considerado. Entretanto, quando analisado o desvio parcial, o método AM1 é superior na maioria das moléculas analisadas, mostrando que o hamiltoniano AM1 não descreve corretamente a conformação das hidroxilas primárias. É importante ressaltar que o método ab initio (HF/3-21G) forneceu resultados semelhantes àqueles obtidos nos níveis semiempíricos.

Ainda com o objetivo de analisar a qualidade das geometrias otimizadas pelos diferentes métodos utilizados, foi feita uma análise das ligações de hidrogênio envolvendo as hidroxilas secundárias. Os parâmetros estruturais utilizados foram as distâncias entre oxigênio...hidrogênio $\left(\mathrm{R}_{\mathrm{OH}}\right)$ e oxigênio...oxigênio $\left(\mathrm{R}_{\mathrm{OO}}\right)$ das hidroxilas secundárias de unidades de glicose adjacentes, bem como o ângulo $\beta$, listado nas Tabelas 1 a 3. Os resultados obtidos são apresentados na Tabela 6, na qual pode ser observada uma redução da distância $\mathrm{O} \ldots \mathrm{O}\left(\mathrm{R}_{\mathrm{OO}}\right)$ e consequiente diminuição da distância $\mathrm{O} . . . \mathrm{H}\left(\mathrm{R}_{\mathrm{OH}}\right)$ da $\alpha$ CD para a $\gamma$-CD. No nível HF/3-21G, os valores de $\mathrm{R}_{\mathrm{OO}}$ e $\mathrm{R}_{\mathrm{OH}}$ calculados para a $\alpha$ e $\gamma$-CD foram, respectivamente, (em $\AA$ ): $3,062,2,112$ e 2,751, 1,793. Esses resultados ab initio são próximos daqueles obtidos no nível PM3 (3,038, 2,234 ( $\alpha$-CD) e 2,732, 1,822 ( $\gamma$-CD)). As distâncias de ligação de hidrogênio calculadas nos níveis MM2 e AM1 foram maiores que aquelas obtidas nos níveis PM3 e HF/321G. De forma geral, a presença de águas de inclusão leva a um aumento das distâncias $\mathrm{R}_{\mathrm{OO}}$ e $\mathrm{R}_{\mathrm{OH}}$. Os resultados PM3 mostram um acordo satisfatório com os dados experimentais, sendo os desvios entre os valores de $\mathrm{R}_{\mathrm{OO}}$ calculados e experimentais menores que $0,1 \AA$. No nível MM2, os resultados relativos às ligações de hidrogênio foram também satisfatórios, exceto para a $\gamma$-CD onde o desvio foi da ordem de $0,3 \AA$. Nas geometrias AM1 os desvios foram mais pronunciados, exceto para a $\alpha-C D$, para a qual um desvio de $0,004 \AA$ foi obtido. De acordo com a literatura ${ }^{23}$, um critério de caracterização de ligações de hidrogênio é possuir uma distância entre os oxigênios menor ou igual a $3,3 \AA\left(\mathrm{R}_{\mathrm{OO}} \leq 3,3 \AA\right)$ e o ângulo $\beta$ menor ou 
Tabela 6. Distâncias interatômicas O...H e O...O (em Å) e ângulo $\beta$ (em graus) envolvendo as hidroxilas secundárias. Os valores apresentados são médias das medidas envolvendo todas as unidades de glicose das ciclodextrinas

\begin{tabular}{|c|c|c|c|c|c|c|c|c|c|c|c|c|}
\hline & \multicolumn{3}{|c|}{ MM2 } & \multicolumn{3}{|c|}{ AM1 } & \multicolumn{3}{|c|}{ PM3 } & \multicolumn{3}{|c|}{$\mathrm{HF} / 3-21 \mathrm{G}$} \\
\hline & $\mathrm{O} \ldots \mathrm{H}$ & О...O & $\beta$ & $\mathrm{O} \ldots \mathrm{H}$ & O...O & $\beta$ & $\mathrm{O} \ldots \mathrm{H}$ & O...O & $\beta$ & $\mathrm{O} \ldots \mathrm{H}$ & O...O & $\beta$ \\
\hline$\alpha-C D$ & 2,417 & 3,153 & 18,9 & 2,488 & 3,236 & 23,1 & 2,234 & 3,038 & 20,0 & 2,112 & 3,062 & 9,6 \\
\hline$\alpha-C D .2 H 2 O$ & 2,465 & 3,190 & 20,3 & 2,501 & 3,226 & 23,8 & 2,565 & 3,266 & 27,2 & - & - & - \\
\hline Raios X & & & & & & & & 3,230 & & & & \\
\hline$\beta-C D$ & 2,016 & 2,902 & 17,2 & 2,202 & 3,044 & 23,6 & 1,813 & 2,726 & 14,1 & 1,796 & 2,772 & 4,1 \\
\hline$\beta-\mathrm{CD} .10 \mathrm{H} 2 \mathrm{O}$ & 2,032 & 2,910 & 16,2 & 2,392 & 3,159 & 28,0 & 1,933 & 2,789 & 18,9 & - & - & - \\
\hline Raios X & & & & & & & & 2,850 & & & & \\
\hline$\gamma-\mathrm{CD}$ & 1,992 & 2,864 & 17,9 & 2,200 & 3,005 & 26,5 & 1,822 & 2,732 & 14,0 & 1,793 & 2,751 & 8,5 \\
\hline$\gamma-\mathrm{CD} .12 \mathrm{H} 2 \mathrm{O}$ & 2,546 & 3,172 & 41,7 & 2,418 & 3,160 & 30,6 & 1,895 & 2,745 & 21,8 & - & - & - \\
\hline Raios X & & & & & & & & 2,823 & & & & \\
\hline
\end{tabular}

igual a $30^{\circ}\left(\beta \leq 30^{\circ}\right)$. Portanto, de acordo com esse critério e os valores da Tabela 6 , pode ser observado que as geometrias obtidas em todos os níveis de teoria contemplam, em média, as ligações de hidrogênio envolvendo as hidroxilas secundárias das moléculas de ciclodextrinas. No nível AM1, as distâncias $\mathrm{R}_{\mathrm{OO}}$ são maiores que $3,0 \AA ̊$ e $\beta$ maior que $23^{\circ}$. Os menores valores para $R_{\mathrm{OO}}$ foram calculados no nível PM3 e os menores valores de $\beta$ no nível HF/3-21G. Considerando os hamiltonianos semi-empíricos AM1 e PM3, os resultados apresentados na Tabela 6 mostram que o método PM3 é superior ao AM1 na descrição de ligações de hidrogênio, fato esse já relatado na literatura ${ }^{8,24}$.

Na última parte do presente estudo, foi realizada uma análise da influência da estrutura no módulo e orientação do momento de dipolo elétrico $(\mu)$ das CD's. Cálculos de $\mu$ foram feitos no nível HF/3-21G considerando as geometrias otimizadas através dos métodos quânticos AM1, PM3 e HF/3-21G. O ângulo de orientação do dipolo $(\chi)$ foi definido em relação ao eixo do tronco de cone (Figura 2b). Os resultados são relatados na Tabela 7. O módulo do dipolo apresentou valores na faixa de $12-18 \mathrm{D}(\alpha-\mathrm{CD}), 6-9 \mathrm{D}(\beta-\mathrm{CD})$ e $13-15 \mathrm{D}(\gamma-\mathrm{CD})$, dependendo da geometria utilizada. Dados experimentais para o momento de dipolo elétrico de ciclodextrinas não foram ainda obtidos, entretanto, valores teóricos relatados na literatura variam de $10-20 D^{15}$. É importante notar o baixo valor de $\mu$ para a $\beta-\mathrm{CD}$, o que pode ser relacionado à baixa solubilidade em água desse análogo ${ }^{15}$.

Tabela 7. Módulo $(\mu)$ e orientação $(\chi)$ do momento de dipolo elétrico calculado para as ciclodextrinas, considerando as estruturas otimizadas nos níveis HF/3-21G, AM1 e PM3

HF/3-21G HF/3-21G//AM1 HF/3-21G//PM3

\begin{tabular}{llccc}
\hline$\alpha$ & $\mu$ /Debye & 17,8 & 11,6 & 15,9 \\
& $\chi$ /graus & 77,6 & 91,9 & 101,4 \\
& & & \\
$\beta$ & $\mu$ /Debye & 6,26 & 7,82 & 8,87 \\
& $\chi$ /graus & 47,7 & 139,3 & 156,4 \\
& & & \\
$\gamma$ & $\mu$ /Debye & 13,4 & 12,5 & 15,1 \\
& $\chi$ /graus & 101,9 & 116,9 & 125,0 \\
\hline
\end{tabular}

A orientação do dipolo é também sensível às distorções estruturais. De maneira geral, a maior contribuição do dipolo está no plano médio da molécula, formado pelos oxigênios anoméricos ${ }^{23}$. Segundo Georg ${ }^{23,}$ esse resultado pode ser analisado em função do fato das estruturas das ciclodextrinas possuírem uma largura equivalente a duas vezes a altura, o que torna mais significativa a contribuição para o dipolo na direção perpendicular ao eixo central do cone, devido às distorções na densidade eletrônica. A diferença mais pronunciada entre as estruturas foi obtida para a $\beta-\mathrm{CD}$, para a qual o momento de dipolo calculado considerando a estrutura HF/3-21G apresentou $\chi=48^{\circ}$, comparado aos valores $139^{\circ}$ (AM1) e $156^{\circ}$ (PM3). Para $\alpha$ e $\gamma$-CD o ângulo $\chi$ foi obtido na faixa de $92-101^{\circ}$ e $117-125^{\circ}$, respectivamente. A orientação do dipolo molecular pode ser importante na determinação da estrutura de complexos de inclusão, podendo afetar o modo de inclusão e a estabilidade do composto formado ${ }^{7}$.

\section{CONCLUSÕES}

As geometrias da $\alpha, \beta$ e $\gamma$-CD foram completamente otimizadas utilizando os métodos de mecânica molecular (MM2), semi-empíricos (AM1 e PM3) e ab initio HF/3-21G. As estruturas obtidas foram comparadas quantitativamente com os dados experimentais disponíveis, através da definição de diferentes parâmetros estruturais. De forma geral, o método MM2 apresentou os menores desvios em relação à estrutura cristalográfica, com valores médios do RMS iguais a $5^{\circ}$ para $\alpha$-CD, $4^{\circ}$ para $\beta-C D$ e $6^{\circ}$ para $\gamma-C D$. No nível ab initio HF/3-21G, os desvios médios foram $6^{\circ}$ para $\alpha, 7^{\circ}$ para $\beta$ e $8^{\circ}$ para $\gamma$-CD's. As estruturas semi-empíricas foram também obtidas em satisfatório acordo com a geometria experimental, com desvios médios calculados para $\alpha, \beta$ e $\gamma$-CD iguais a $6^{\circ}$ (AM1) e $7^{\circ}$ (PM3), $7^{\circ}$ (AM1) e $6^{\circ}$ (PM3), $7^{\circ}$ (AM1) e $6^{\circ}$ (PM3), respectivamente. De forma sistemática, os desvios foram mais pronunciados quando consideradas as águas de inclusão. Os principais parâmetros estruturais responsáveis pelos desvios calculados são os ângulos diedros $\Omega$ e $\Xi$, os quais definem a conformação das hidroxilas primárias, sendo os erros na descrição desse parâmetro mais pronunciados no método AM1.

Considerando os métodos semi-empíricos, as principais diferenças estão associadas à descrição das ligações de hidrogênio envolvendo as hidroxilas secundárias das CD's. Observou-se, por exemplo, que para a $\beta$-CD as distâncias médias entre oxigênios adjacentes $\left(\mathrm{R}_{\mathrm{OO}}\right)$ foram calculadas iguais a 2,8 (HF/3-21G), 2,8 (PM3), 3,0 (AM1) e 2,9 ̊ (MM2). O valor médio observado no estado sólido é de 2,9 Å. As distâncias de ligação de hidrogênio $\mathrm{H}$...O $\left(\mathrm{R}_{\mathrm{OH}}\right)$ foram 1,8 (PM3) e 2,2 $\AA$ (AM1), mostrando a superioridade do método PM3 na descrição de ligações de hidrogênio intramoleculares. Esse resultado também foi observado para as outras CD's estudadas. Portanto, os resultados do presente trabalho mostram que os métodos clássicos e quânticos fornecem resultados satisfatórios considerando as estruturas das ciclodextrinas, sendo mecânica molecular (MM2) o mais indicado. No nível quântico, os resultados semi-empíricos 
(AM1 e PM3) foram equivalentes ao ab initio $\mathrm{HF} / 3-21 \mathrm{G}$, com o hamiltoniano PM3 fornecendo melhores resultados, principalmente em relação à descrição de ligações de hidrogênio intramoleculares. É importante ressaltar que a análise estrutural desenvolvida no presente artigo foi feita comparando as geometrias teóricas isoladas com dados experimentais obtidos no estado sólido. Os resultados de estudos dessa natureza podem levar a conclusões que não refletem a capacidade de métodos teóricos puros em reproduzir estruturas moleculares, em função da diferença de ambiente no qual a molécula se encontra. Entretanto, dois aspectos são relevantes: (i) métodos de cálculo empíricos e semi-empíricos possuem parâmetros ajustados para reproduzir propriedades moleculares obtidas em fase condensada, portanto, parte dos efeitos causados pelo meio está implicitamente incluída nesses métodos e (ii) dados experimentais, principalmente estruturas de moléculas como as ciclodextrinas, estão disponíveis apenas em fase condensada, tornando os estudos teóricos comparativos restritos a esse conjunto de informações.

\section{AGRADECIMENTOS}

Os autores agradecem à Fundação de Amparo à Pesquisa do Estado de Minas Gerais (FAPEMIG) pelo auxílio concedido para o desenvolvimento desse projeto.

\section{REFERÊNCIAS}

1. Villers, A.; Acad. Sci. Paris 1891, 112, 536.

2. Sundararajan, P. R.; Rao, V. S. R.; Carbohydr. Res. 1970, 13, 351.
3. French, A. D.; Murphy, V. G.; Carbohydr. Res. 1973, 27, 391.

4. French, A. D.; Murphy, V. G.; Polymer 1977, 18, 489.

5. Jicsinszky, L.; Bakó, I.; J. Inclusion Phenom. Macrocyclic Chem. 1994, $18,275$.

6. Botsi, A.; Yannakopoulou, K.; Hadjoudis, E.; Waite, J.; Carbohydr. Res. 1996, 283, 1 .

7. Lipkowitz, K. B.; Chem. Rev. 1998, 98, 1829.

8. Li, X. S.; Liu, L.; Monatsh. Chem. 2000, 131, 849

9. Bodor, N.; Buchwald, P.; J. Inclusion Phenom. Macrocyclic Chem. 2002, 44,9 .

10. Barbiric, D. J.; de Rossi, R. H.; Castro, E. A.; J. Mol. Struc.- Theochem. 2001, 537, 235.

11. Liu, L.; Li, X. S.; Guo, Q. X.; J. Mol. Struc.- Theochem. 2000, 530, 31.

12. Cervelló, E.; Mazzucchi, F.; Jaime, C.; J. Mol. Struc. 2000, 530, 155.

13. Dos Santos, H. F.; Duarte, H. D.; Sinisterra, R. D.; De Melo-Mattos, S. V.; De Oliveira, L. F. C.; De Almeida, W. B.; Chem. Phys. Lett. 2000, 319 , 569.

14. http://www.ccdc.cam.ac.uk/, acessada em Julho 2003.

15. Connors, K. A.; Chem. Rev. 1997, 97, 1325.

16. Manor, P. C.; Seanger, W.; J. Am. Chem. Soc. 1974, 96, 3630.

17. Zabel, V.; Saenger, W.; Mason, S. A.; J. Am. Chem. Soc. 1986, 108, 3664.

18. Linder, K.; Saenger, W.; Carbohydr. Res.; 1982, 99, 103.

19. Heyes, S. J.; Clayden, N. J.; Dobson, C. M.; Carbohydr. Res. 1992, 283, 1.

20. Wavefunction, Inc.; PC Spartan Pro; Irvine, Estados Unidos da América, 1999.

21. Frisch, M. J.; et al.; GAUSSIAN 98; Pittsburgh, Estados Unidos da América, 1998.

22. Cambridge Soft Corporation; CS Chem 3 D Pro; Cambridge, Estados Unidos da América, 1997.

23. Georg, H. C.; Dissertação de Mestrado, Universidade de São Paulo, Brasil, 2002.

24. Li, X. S.; Liu, L.; Gou, Q. X.; Xhou, S. D.; Liu, Y. C.; Chem. Phys. Lett. 1999, 307, 117. 